Check for updates

Cite this: RSC Adv., 2017, 7, 54266

Received 27th September 2017 Accepted 17th November 2017

DOI: $10.1039 / c 7 r a 10697 a$

rsc.li/rsc-advances

\section{Phosphomolybdic acid immobilized on ionic liquid- modified hexagonal boron nitride for oxidative desulfurization of fuel $\uparrow$}

\author{
Haiyan Ji, ${ }^{a}$ Haitao Ju, ${ }^{a}$ Rong Lan, ${ }^{b}$ Peiwen Wu, (D) ${ }^{c}$ Jia Sun, ${ }^{a}$ Yanhong Chao, (D) ${ }^{b}$ \\ Suhang Xun, ${ }^{b}$ Wenshuai Zhu iD *b and Huaming $\mathrm{Li}^{\mathrm{d}}$
}

\begin{abstract}
Hexagonal boron nitride supported catalysts have been well-developed in catalytic desulfurization, but struggle from the drain of catalytically active sites. Herein, an ionic liquid functionalized BN is constructed for stabilization of catalytic sites (phosphomolybdic acid, HPMo). A stable and welldispersed supported catalyst was successfully obtained, which was confirmed by a series of characterizations. Detailed oxidative desulfurization experiments showed that the IL-induced high dispersity of the HPMo indicated a promising catalytic performance; meanwhile, the IL-assisted stability dominated an enhanced recycling performance. Additionally, the satisfying catalyst exhibited an excellent catalytic activity to numerous sulfur compounds and a fine resistance to interferents. Moreover, the possible reaction process was studied by GC-MS.
\end{abstract}

\section{Introduction}

In recent years, fossil fuels have been widely used in various fields, and $80 \%$ of energy around the world originates from fossil fuels. However, the sulfur content has turned to be a barrier to the quality of crude oil. ${ }^{\mathbf{1} 2}$ Owing to the increasingly serious environmental pollution, regulations have been proposed by many countries to limit the sulfur content in fuel oils to not exceeding $10 \mathrm{ppm} .^{3-5}$ Hence, numerous efforts have been devoted to seeking a more efficient method for reducing the sulfur content in fuel oils. Traditionally, the hydrodesulfurization (HDS) method has been industrially employed but struggles from many disadvantages including.6-8 harsh reaction conditions and poorer removal efficiencies of aromatic organic sulfides, such as dibenzothiophene (DBT), 4,6dimethyldibenzothiophene (4,6-DMDBT), etc. More recently, a milder desulfurization process, oxidative desulfurization (ODS), which shows excellent performance in the oxidation of aromatic sulfur compounds, have been developed. ${ }^{6-9-13}$ The oxidative desulfurization process involves oxidation of sulfur compounds for an enhanced polarity and further adsorptive or extractive removal. ${ }^{14-16}$

${ }^{a}$ School of Materials Science and Engineering, Jiangsu University, Zhenjiang 212013, China

${ }^{b}$ School of Chemistry and Chemical Engineering, Jiangsu University, Zhenjiang 212013, China. E-mail: zhuws@ujs.edu.cn

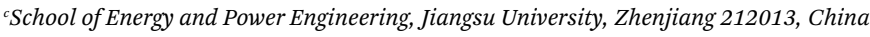
${ }^{d}$ Institute for Energy Research, Jiangsu University, Zhenjiang 212013, China

$\dagger$ Electronic supplementary information (ESI) available. See DOI: $10.1039 / \mathrm{c} 7 \mathrm{ra} 10697 \mathrm{a}$
In terms of the sulfur oxidation process, numerous reports have demonstrated that heteropoly acid (HPA) and its derivatives are a branch of high-efficiency catalysts. ${ }^{17-19}$ Although some advantages of HPAs, including strong Brönsted acid, oxidation-reduction, and structural selectivity properties, can be expected, numerous shortages are still inevitable. For example, low specific surface areas, poor stability, and dissolution property. ${ }^{20,21}$ To avoid the disadvantages, loading HPAs onto a certain support has been proved to be an effective strategy for high catalytic activity and excellent stability. ${ }^{22-24}$

Among all current supports, hexagonal boron nitride (h-BN) is one of the most favorable ones because of the high specific surface areas, layered structure, excellent thermal stability and so on. ${ }^{25-33}$ However, all current reported h-BN supported catalysts were gained by impregnation process, leading to the poor dispersion and agglomeration during the catalytic performance, especially in liquid phase reactions such as oxidative desulfurization, leading to the poorer recycling performance. Some more advanced supporting methods are thusly called. In colloid chemistry, long organic chains are often employed as media to prevent the aggregation of active sites. ${ }^{3,35}$ Additionally, HPAs are proved can be uniformly dispersed in organic media, such as ionic liquids. Further inspired by the nature of h-BN, which holds numerous functional groups on its surface, which can be modified as functional sites for grafting ionic liquids, further disperse the HPAs uniformly.

In this work, the graphene-like h-BN was functionalized by ionic liquids (BN-IL) and further employed as a functional support for dispersion of phosphomolybdic acid $\left(\mathrm{H}_{3} \mathrm{PMO}_{12} \mathrm{O}_{40}, \mathrm{HPMo}\right)$ to obtain a highly-active catalyst (HPMo/BN-IL). Owing to the functionalized property of the BN-IL, both high dispersion and 
excellent stability of the supported HPMo can be expected. The asprepared heterogeneous catalyst was further characterized detailedly. The obtained HPMo/BN-IL was further applied in oxidative desulfurization process, showing a promising performance in catalytic oxidation of dibenzothiophene (DBT), 4,6-dimethyldibenzothiophene (4,6-DMDBT) and 3-methylbenzothiophene (3-MBT). Because of the enhanced stability, a better recycling performance was acquired, compared with that without IL functionalization. The current work would provide a new strategy for preparation of h-BN supported catalysts.

\section{Experimental section}

\subsection{Materials}

$N$-Methylimidazole $\left(\mathrm{C}_{4} \mathrm{H}_{6} \mathrm{~N}_{2}\right.$, C.P. grade $)$, anhydrous diethyl ether $\left(\mathrm{C}_{4} \mathrm{H}_{10} \mathrm{O}\right.$, A.R. grade), diboron trioxide $\left(\mathrm{B}_{2} \mathrm{O}_{3}\right.$, A.R. grade), urea $\left(\mathrm{CO}\left(\mathrm{NH}_{2}\right)_{2}\right.$, A.R. grade), toluene $\left(\mathrm{C}_{6} \mathrm{H}_{5} \mathrm{CH}_{3}\right.$, A.R. grade), dichloromethane $\left(\mathrm{CH}_{2} \mathrm{Cl}_{2}, \mathrm{~A} . \mathrm{R}\right.$. grade), anhydrous ethanol $\left(\mathrm{C}_{2} \mathrm{H}_{5} \mathrm{OH}\right.$, A.R. grade), phosphomolybdic acid $\left(\mathrm{H}_{3} \mathrm{PMo}_{12} \mathrm{O}_{40}\right.$, aliased as HPMo, A.R. grade), $n$-octane $\left(\mathrm{C}_{8} \mathrm{H}_{18}\right.$, A.R. grade), tetradecane $\left(n-\mathrm{C}_{14} \mathrm{H}_{30}, 99 \%\right)$, hydrogen peroxide $\left(\mathrm{H}_{2} \mathrm{O}_{2}, 30 \mathrm{wt} \%\right)$, cyclohexene $\left(\mathrm{C}_{6} \mathrm{H}_{10}\right.$, A.R. grade), paraxylene $\left(\mathrm{C}_{6} \mathrm{H}_{4}\left(\mathrm{CH}_{3}\right)_{2}\right.$, A.R. grade), acetone $\left(\mathrm{CH}_{3} \mathrm{COCH}_{3}\right.$, A.R. grade), commercial bulk boron nitride (aliased as commercial-BN, 98\%) were derived from Shanghai Sinopharm Chemical Reagent Co., Ltd. (3-Chloropropyl) trimethoxysilan $\left(\mathrm{C}_{6} \mathrm{H}_{15} \mathrm{ClO}_{3} \mathrm{Si}\right.$, 98\%) was purchased from Aladdin Chemistry. 4,6-Dimethyldibenzothiophene (4,6-DMDBT, 97\%), dibenzothiophene (DBT, 98\%), benzothianaphthene (BT, 99\%) and 3-methylbenzothiophene (3-MBT, 96\%) were marketed by Sigma-Aldrich.

\subsection{Preparation process of catalysts}

2.2.1 Synthesis of ionic liquids (IL). $10.3160 \mathrm{~g}(0.1258 \mathrm{~mol})$ of freshly distilled $N$-methylimidazole and $25 \mathrm{~g}(0.1258 \mathrm{~mol})$ of (3-chloropropyl)trimethoxysilan were added into a roundbottom flask and refluxed at $95{ }^{\circ} \mathrm{C}$ for $24 \mathrm{~h}$ with continuous stirring. After being cooled to room temperature, the obtained viscous liquid is washed with anhydrous diethyl ether and then transferred to a vacuum oven at $40{ }^{\circ} \mathrm{C}$ for $12 \mathrm{~h}$. The collected faint yellow viscous liquid is the prepared ionic liquids (IL).

2.2.2 Preparation of h-BN. $0.6962 \mathrm{~g}(0.01 \mathrm{~mol})$ of $\mathrm{B}_{2} \mathrm{O}_{3}$ and $12.012 \mathrm{~g}(0.2 \mathrm{~mol})$ of $\mathrm{CO}\left(\mathrm{NH}_{2}\right)_{2}$ are added to a beaker with $40 \mathrm{~mL}$ of ultrapure water. Then the beaker with mixture was transferred to an isothermally heated oil bath at $60{ }^{\circ} \mathrm{C}$ with continuous stirring to completely evaporate the solvent. Afterwards, the obtained white crystal was transferred into a tube furnace, and the calcining process was as follows: the crystal mixtures are thermally treated from room temperature to $550{ }^{\circ} \mathrm{C}$ with a heating ratio of $5{ }^{\circ} \mathrm{C} \mathrm{min}^{-1}$ and then kept for $120 \mathrm{~min}$; then the tube furnace was further treated from $550{ }^{\circ} \mathrm{C}$ to $1000{ }^{\circ} \mathrm{C}$ at $5{ }^{\circ} \mathrm{C} \min ^{-1}$ and kept for another $120 \mathrm{~min}$. The tube furnace was protected by $\mathrm{N}_{2}$ atmosphere during calcining process. After cooling to room temperature, the white products are collected and denoted as h-BN

2.2.3 Preparation of BN-IL. $1.0 \mathrm{~g}$ of $\mathrm{h}-\mathrm{BN}$ and $0.5 \mathrm{~g}$ of IL are added to a round-bottom flask and dispersed into $50 \mathrm{~mL}$ of toluene. Then the flask with mixtures was refluxed at $90{ }^{\circ} \mathrm{C}$ for $16 \mathrm{~h}$ with continuous stirring. After cooling to room temperature, the mixtures are filtrated and then washed by $\mathrm{CH}_{2} \mathrm{Cl}_{2}$ to remove the unreacted IL to get white products. Finally, the white products are put into a vacuum oven at $60{ }^{\circ} \mathrm{C}$ for $12 \mathrm{~h}$. The obtained white powder is the ionic liquid-modified h-BN, which denoted as BN-IL.

2.2.4 Preparation of HPMo/BN-IL catalyst. $0.04 \mathrm{~g}$ of HPMo was dissolved in $20 \mathrm{~mL}$ of anhydrous ethanol with stirring for $30 \mathrm{~min}$, then $0.16 \mathrm{~g}$ of BN-IL was also added and stirred for $8 \mathrm{~h}$. Then the mixture was washed with anhydrous ethanol and dried by a dryer at $100{ }^{\circ} \mathrm{C}$ for $12 \mathrm{~h}$. The obtained faint yellow powder is the prepared HPMo/BN-IL catalyst with $20 \mathrm{wt} \%$ of loading amounts, which denoted as $20 \% \mathrm{HPMo} / \mathrm{BN}-\mathrm{IL}$. Other $x \% \mathrm{HPMo} / \mathrm{BN}-\mathrm{IL}$ catalysts $(x=5,10,30)$ were prepared by the same process, just changed the amount of HPMo.

The preparation process of $\mathrm{HPMo} /$ commercial-BN-IL is similar to that of HPMo/BN-IL catalyst, merely replacing the h-BN with commercial-BN.

2.2.5 Preparation of HPMo/BN catalyst. $0.04 \mathrm{~g}$ of HPMo was dissolved in $20 \mathrm{~mL}$ of anhydrous ethanol with stirring for $30 \mathrm{~min}$, then $0.16 \mathrm{~g}$ of $\mathrm{h}-\mathrm{BN}$ was also added and stirred for $8 \mathrm{~h}$ to fully disperse. Then the mixture was washed with anhydrous ethanol and dried at $100{ }^{\circ} \mathrm{C}$ for $12 \mathrm{~h}$. The obtained white powder is the prepared HPMo/BN catalyst with $20 \%$ (wt $\%$ ) of loading amounts for HPMo, which denoted as $20 \% \mathrm{HPMo} / \mathrm{BN}$.

\subsection{Characterization}

X-ray diffraction (XRD) micrographs of all samples were analyzed by a D8 Advanced X-ray diffraction to detect crystal structures of samples, which was operated with $\mathrm{Cu} \mathrm{K} \alpha$ radiation. Using a Hitachi H-700 transmission electron microscope to record transmission electron microscopy (TEM) images to examine structures and morphologies of various samples. All samples were conducted by a Nicolet Nexus 470 Fourier transform infrared spectrometer, which is equipped with a $\mathrm{KBr}$ pellets at room temperature, to receive the Fourier transform infrared spectroscopy (FT-IR) spectra. An ESCALAB250 (Thermo VG, UK) system with $1486.6 \mathrm{eV}$ of standard monochromatic Al $\mathrm{K} \alpha$ excitation was used to analyze X-ray photoelectron spectroscopy (XPS) spectra. Structural information of samples was exhibited in Raman spectra, which was acquired from a Thermo Scientific DXR Smart Raman spectrometer with a $532 \mathrm{~nm}$ excitation. A Shimadzu UV-2450 spectrophotometer was operated to measure ultraviolet-visible diffuse reflectance spectroscopies (UV-vis DRS) of samples, which was run with a spherical diffuse reflectance accessory. A TriStar II 3020 surface-area and porosity analyzer were implemented to test nitrogen adsorptiondesorption isotherms and pore-size distribution curves of various samples. Gas chromatography-mass spectrometry (GC-MS) was performed to observe products and mechanism of the catalytic system.

\subsection{Oxidative desulfurization process}

2.4.1 Preparation of model oil. The preparation process of 4,6-DMDBT, DBT, 3-MBT and BT model oil: a certain amount of 
4,6-DMDBT, DBT, 3-MBT, and BT were respectively dissolved in $n$-octane with sulfur contents of $500 \mathrm{ppm}$, which metered volume by tetradecane. And the corresponding concentrations of internal standard were 1000 ppm, 4000 ppm, 1000 ppm and 500 ppm, respectively.

The preparation process of interferential model oil for 4,6DMDBT: a certain amount of cyclohexene, methylbenzene, and paraxylene were respectively dissolved in 4,6-DMDBT model oil to obtain cyclohexene interference oil, methylbenzene interference oil and paraxylene interference oil. And the corresponding sulfur content and internal standard concentrations are $500 \mathrm{ppm}$ and $1000 \mathrm{ppm}$, respectively.

The preparation process of mixed interferential model oil for 4,6-DMDBT: a certain amount of cyclohexene, methylbenzene, and paraxylene are simultaneously dissolved in 4,6-DMDBT model oil to obtain mixed interferential model oil. And the corresponding sulfur content and internal standard concentration are also $500 \mathrm{ppm}$ and $1000 \mathrm{ppm}$, respectively.

2.4.2 Oxidative desulfurization. The reaction equipment of oxidation desulfurization for model oil was carried out in a home-made two-necked flask. In turn, $0.05 \mathrm{~g}$ of catalyst and $5 \mathrm{~mL}$ of model oil were added into a two-necked flask, and a certain amount of $30 \mathrm{wt} \% \mathrm{H}_{2} \mathrm{O}_{2}$ was also added after stirring. The reaction was operated in a thermostatic water bath with constant stirring. During the process of reaction, the upper oil phase was periodically withdrawn, and $1 \mu \mathrm{L}$ of oil phase was injected into a gas chromatography detector to detect the residual sulfur content. Other active experiments were operated by a similar process, only changing reaction factors. The residual sulfur content was used to calculate removal rate of sulfide, which represents the activity of the catalyst in ODS.

Residual sulfur content is measured by a gas chromatographyflame ionization detector (GC-FID, Agilent 7890A, HP-5 column, $30 \mathrm{~m}$ long $\times 0.32 \mathrm{~mm}$ inner diameter (id) $0.25 \mu \mathrm{m}$ film thickness), using tetradecane as an internal standard.

\section{Results and discussion}

\subsection{X-ray diffraction analysis}

The X-ray diffraction (XRD) patterns of h-BN, BN-IL, HPMo and $x \% \mathrm{HPMo} / \mathrm{BN}-\mathrm{IL}$ catalysts are displayed in Fig. 1 . As seen from Fig. 1a, two obvious characteristic peaks for the hexagonal phase of $\mathrm{BN}$ at $2 \theta=23.8^{\circ}$ and $41.9^{\circ}$ were clearly detected, respectively attributable to the (002) and (100) lattice planes (JCPDS Card no. 34-0421). ${ }^{36-38}$ The two peaks were also found in BN-IL (Fig. 1b), indicating the stability of h-BN during the IL grafting process. Also, the stability property also existed within the loading of HPMo onto BN-IL (Fig. 1c-f). No characteristic peaks for HPMo were detected in $\mathrm{HPMo} / \mathrm{BN}-\mathrm{IL}$, which is assigned to the low loading amount and high dispersion of HPMo on BN-IL.

\subsection{TEM and EDS-mapping analysis}

In order to study morphology and structure information of HPMo on the carrier, the transmission electron morphologies (TEM) of h-BN and 20\% HPMo/BN-IL catalyst were performed in Fig. 2. TEM images of h-BN in Fig. $2 a$ and $b$ show that h-BN was

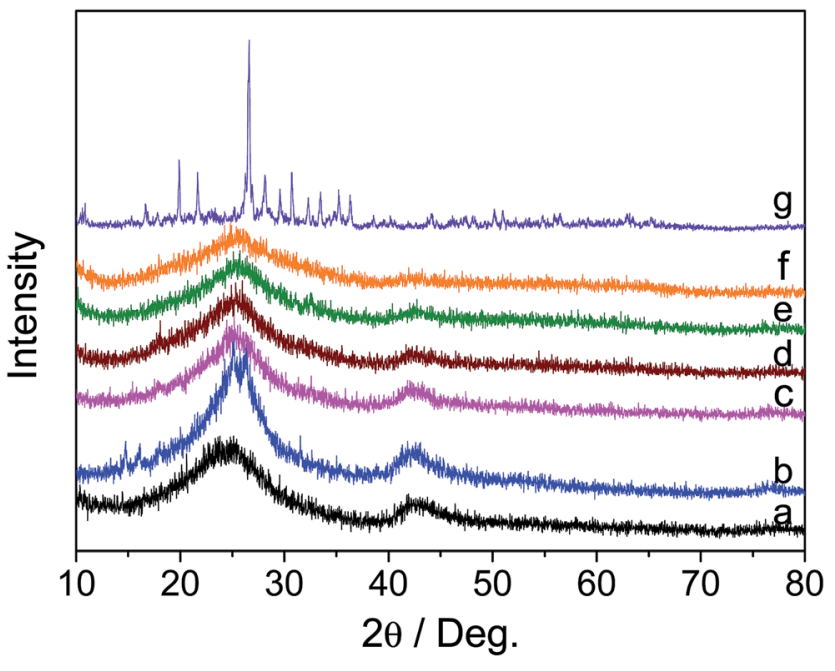

Fig. 1 XRD patterns of all samples. (a) h-BN; (b) BN-IL; (c) $5 \% \mathrm{HPMo} /$ $\mathrm{BN}-\mathrm{IL}$; (d) $10 \% \mathrm{HPMo} / \mathrm{BN}-\mathrm{IL}$; (e) $20 \% \mathrm{HPMo} / \mathrm{BN}-\mathrm{IL}$; (f) $30 \% \mathrm{HPMo} /$ BN-IL; (g) HPMo.

a layered structure with numerous pores. As can be seen from Fig. $2 \mathrm{c}$ and d, the lamellar structure and porous structure of h$\mathrm{BN}$ were retained without damage in HPMo/BN-IL after immobilization, demonstrating the stability of h-BN in the preparation process. Notably, with the presence of HPMo, no clear section for HPMo was detected in the TEM image indicating the uniform dispersion of the HPMo with the grafting of ILs on BN. The similar conclusion can be also obtained from the (Energy Disperse Spectroscopy) EDS mapping characterization in Fig. $\mathrm{S} 1, \dagger$ that the Mo element is uniformly dispersed.

\subsection{FT-IR characterization}

To further verify the immobilization process, the FT-IR spectra of all samples are shown in Fig. 3. Fig. 3a showed the FT-IR spectra of IL, h-BN, BN-IL, HPMo/BN-IL, and HPMo. As can be seen from Fig. $3 \mathrm{a}$, the prepared h-BN exhibited two strong absorption peaks at $804 \mathrm{~cm}^{-1}$ and $1383 \mathrm{~cm}^{-1}$, which belong to the $\mathrm{B}-\mathrm{N}-\mathrm{B}$ stretching vibration mode and in-plane $\mathrm{B}-\mathrm{N}$ transverse optional mode of $\mathrm{h}$ $\mathrm{BN},{ }^{37,39}$ respectively. In the FT-IR spectrum of BN-IL sample, besides the absorption peaks of h-BN, some peaks of IL were also detected (Fig. 3b). The peaks around $1063 \mathrm{~cm}^{-1}$ and $1190 \mathrm{~cm}^{-1}$ are consistent with in-plane $\mathrm{C}-\mathrm{H}$ absorption peaks of the benzene ring, and the $2843 \mathrm{~cm}^{-1}$ and $2947 \mathrm{~cm}^{-1}$ are assigned to $\mathrm{C}-\mathrm{H}$ stretching absorption peaks, ${ }^{\mathbf{8} 40}$ showing that the IL had been successfully immobilized onto h-BN. Moreover, the characteristic peaks for the Keggin structure of HPMo were detected at $789 \mathrm{~cm}^{-1}$ (Mo- $\mathrm{O}_{\mathrm{c}}-\mathrm{Mo}$ corner sharing), $870 \mathrm{~cm}^{-1}$ ( $\mathrm{Mo}-\mathrm{O}_{\mathrm{b}}-\mathrm{Mo}$ edge sharing), $962 \mathrm{~cm}^{-1}$ (P-O-P vibration mode) and $1065 \mathrm{~cm}^{-1}\left(\mathrm{Mo}-\mathrm{O}_{\mathrm{d}}\right.$ vibration mode), ${ }^{4-43}$ respectively. Obviously, the main absorption peaks of h-BN and partial peaks of IL and HPMo were also observed on HPMo/BN-IL catalyst, indicating the successful immobilization of HPMo on BN-IL carrier. However, owing to the cover of $804 \mathrm{~cm}^{-1}$ peaks in h-BN, the $789 \mathrm{~cm}^{-1}$ peak of HPMo was not detected on HPMo/BN-IL. In addition, for HPMo/BN-IL, the 

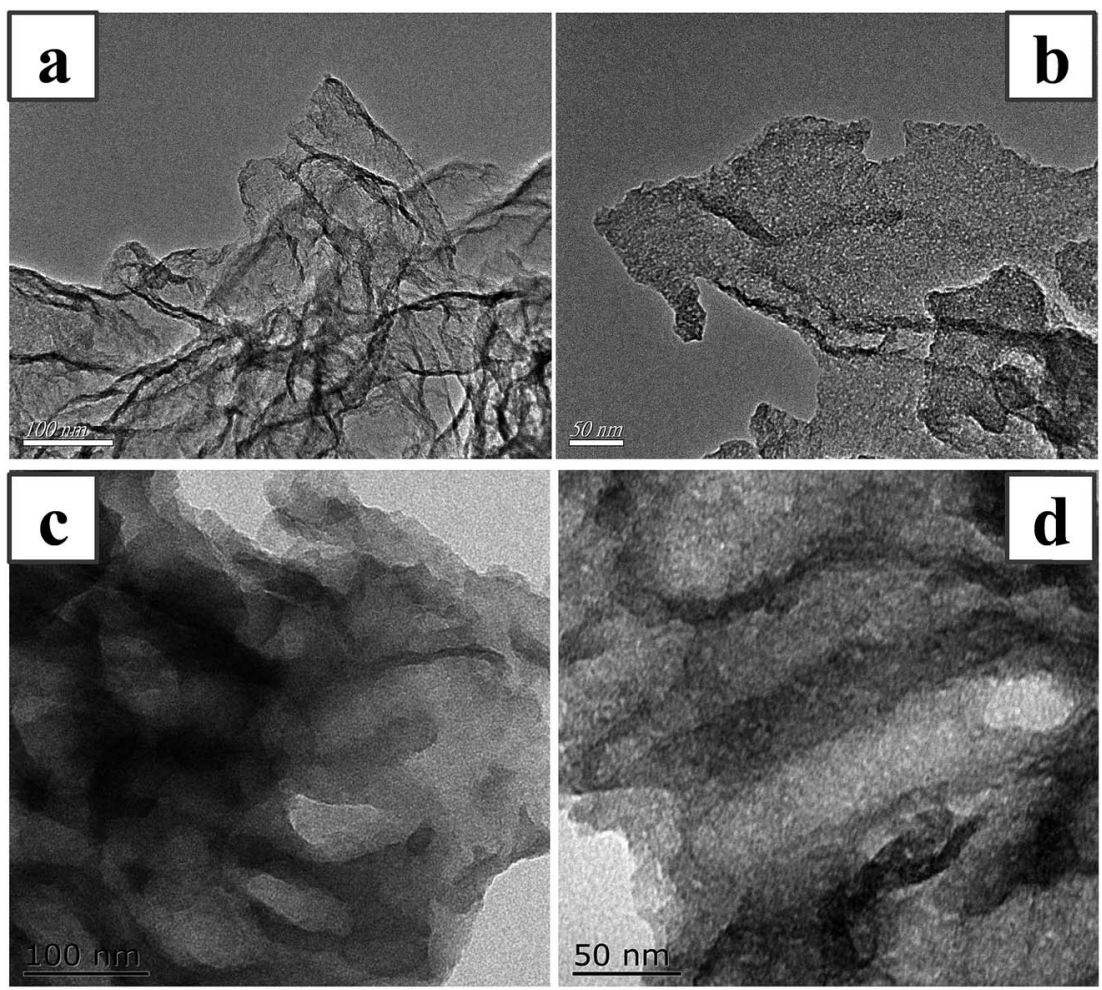

Fig. 2 TEM images of samples. (a, b): h-BN; (c, d): 20\% HPMo/BN-IL.

$870 \mathrm{~cm}^{-1}$ peak of HPMo shifted to higher wavenumber (Fig. 3c), which is attributed to the interaction between HPMo and BN-IL.

\subsection{X-ray photoelectron spectroscopy}

The chemical composition of catalysts was further analyzed by Xray photoelectron spectroscopy (XPS) in Fig. 4. As seen from
Fig. 4a, the HPMo/BN-IL was composed of O, C, N, B, Mo, P and Si elements, showing that the HPMo and IL were successfully introduced onto h-BN. Fig. 4b-e are the high-resolution spectra of $\mathrm{C} 1 \mathrm{~s}, \mathrm{O} 1 \mathrm{~s}, \mathrm{~N} 1 \mathrm{~s}$ and $\mathrm{B} 1 \mathrm{~s}$ core level. The $\mathrm{C} 1 \mathrm{~s}$ core level was divided into three peaks at $284.5 \mathrm{eV}, 286.1 \mathrm{eV}$ and $288.2 \mathrm{eV}$, corresponding to $\mathrm{C}-\mathrm{C}, \mathrm{C}=\mathrm{N}$ and $\mathrm{C}-\mathrm{N}$ state, ${ }^{44,45}$ respectively. The $\mathrm{O}$ 1s core level was separated into three peaks, locating at 530.1, 531.3, and
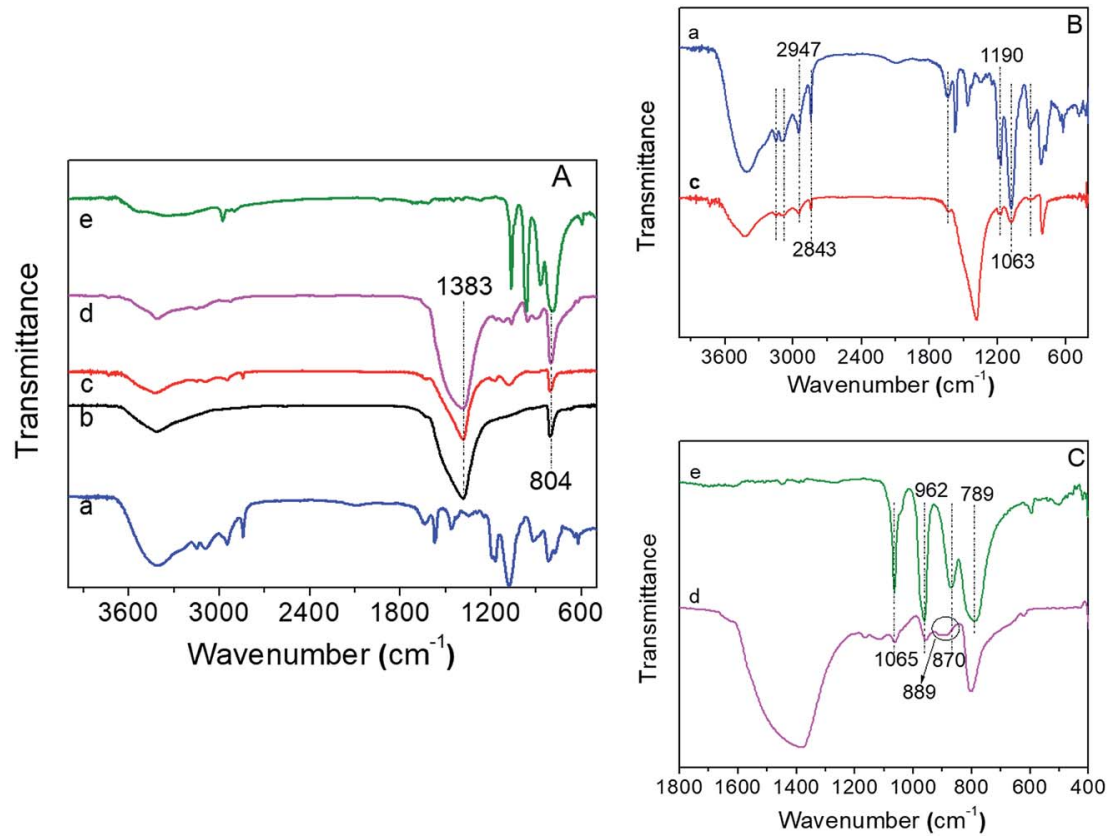

Fig. 3 FT-IR patterns of all samples. (a) IL; (b) h-BN; (c) BN-IL; (d) HPMo/BN-IL; (e) HPMo. 


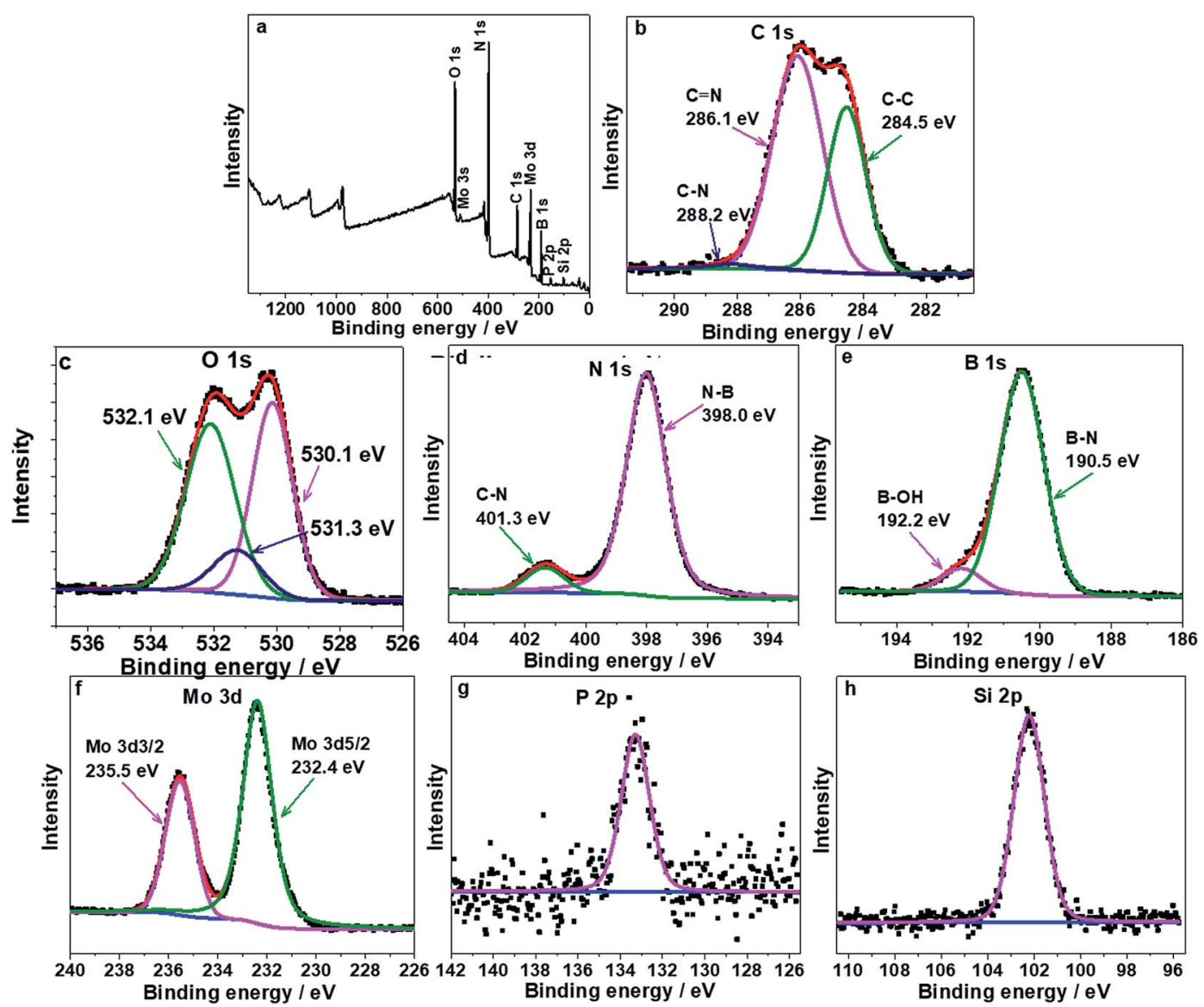

Fig. 4 XPS spectra of (a) survey, (b) C 1s core level, (c) O 1s core level, (d) N 1s core level, (e) B 1s core level, (f) Mo 3d core level, (g) P 2p and (h) Si $2 p$ core level in $20 \%$ HPMo/BN-IL catalyst.

$532.1 \mathrm{eV}$, respectively attribute to $\mathrm{Mo}-\mathrm{O}, \mathrm{O}^{2-}$ for $\mathrm{HPMo}$ and $-\mathrm{OH}$ groups on the surface of h-BN., ${ }^{\mathbf{4 6}-48}$ respectively. The $\mathrm{N} 1 \mathrm{~s}$ peak in Fig. 4d could be separated into $\mathrm{N}-\mathrm{B}$ and $\mathrm{C}-\mathrm{N}$ state, and the binding energies of them were $398.0 \mathrm{eV}$ and $401.3 \mathrm{eV}$ (ref. 37 and 44), respectively. Fig. 4e shows that the B 1 s peak can be divided into B-N (190.5 eV) and B-OH (192.2 eV) state. ${ }^{37,49}$ Compared to the $\mathrm{N}$ 1s and $\mathrm{B} 1 \mathrm{~s}$ core level of $\mathrm{h}-\mathrm{BN}$ in reported literature, the current prepared $\mathrm{BN}$ is a hexagonal phase structure. ${ }^{\mathbf{4 4 0} 50}$ Fig. $4 \mathrm{f}$ is the high-resolution spectra of Mo 3d core level. After a careful comparison with the previous report, the peaks for Mo $5 \mathrm{~d}$ around $232.40 \mathrm{eV}$ and $235.6 \mathrm{eV}$ are respectively attributed to Mo $3 \mathrm{~d}_{5 / 2}$ and Mo $3 \mathrm{~d}_{3 / 2}$, and the valence state of Mo is $a+6$. The result shows that the HPMo is rather stable during the loading process., ${ }^{51-54}$ respectively. Fig. $4 \mathrm{~g}$ and $\mathrm{h}$ are the high-resolution spectra of $\mathrm{P} 2 \mathrm{p}$ and Si 2p core level, the binding energies of them were $133.3 \mathrm{eV}$ and $102.2 \mathrm{eV}$, respectively. The analysis results of XPS spectra are consistent with the results in FT-IR spectra.

\subsection{Raman analysis}

Other structural information of catalysts was further obtained from Raman spectra in Fig. 5. As seen from Fig. 5, an obvious peak for h-BN is detected around $1372.9 \mathrm{~cm}^{-1}$, which belongs to the $\mathrm{B}-\mathrm{N}$ tangential vibration mode $\left(\mathrm{E}_{2 \mathrm{~g}}\right)$ of $\mathrm{h}-\mathrm{BN},{ }^{55}$ showing the hexagonal phase structure. The $\mathrm{E}_{2 \mathrm{~g}}$ peak of h-BN was still observed on the BN-IL and $x \%$ HPMo/BN-IL catalysts, which illustrated that the structure of h-BN was not destroyed after the introduction of HPMo and IL. The peaks of IL at $2841.8 \mathrm{~cm}^{-1}$

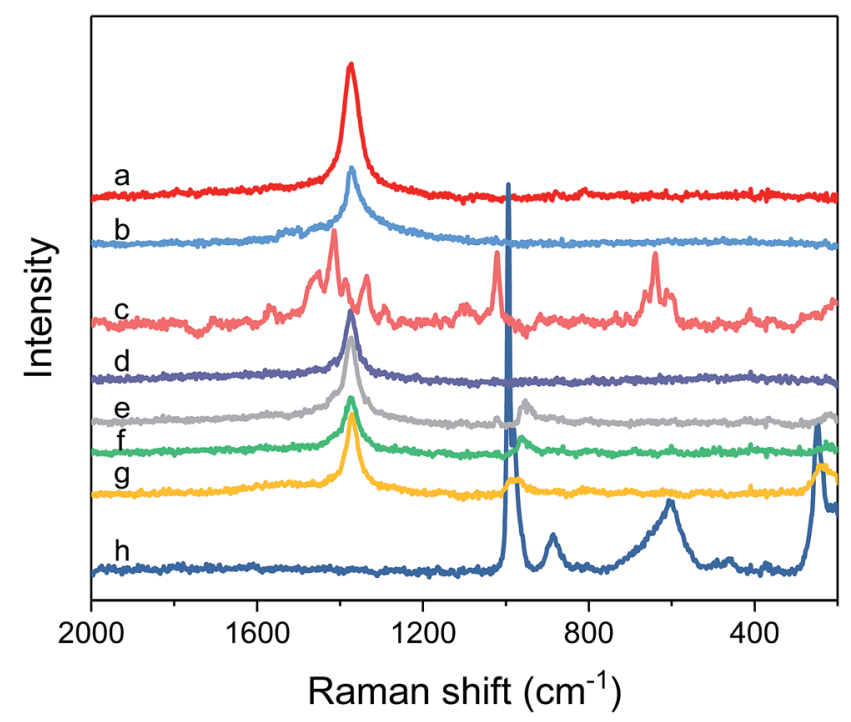

Fig. 5 Raman spectra of all catalysts. (a) h-BN; (b) BN-IL; (c) IL; (d) $5 \%$ $\mathrm{HPMO} / \mathrm{BN}-\mathrm{IL}$; (e) $10 \% \mathrm{HPMO} / \mathrm{BN}-\mathrm{IL}$; (f) $20 \% \mathrm{HPMO} / \mathrm{BN}-\mathrm{IL}$; (g) $30 \%$ HPMO/BN-IL; (h) HPMo. 
and $2950.7 \mathrm{~cm}^{-1}$ were observed in the Raman image of BN-IL. The results state that the IL was successfully introduced onto the surface of $\mathrm{h}-\mathrm{BN}$. The $x \% \mathrm{HPMo} / \mathrm{BN}-\mathrm{IL}$ catalysts also exhibited the characteristic peaks of IL, indicating the existence of IL on catalysts. Characteristic peaks of HPMo were detected around $994 \mathrm{~cm}^{-1}, 886 \mathrm{~cm}^{-1}$ and $599 \mathrm{~cm}^{-1}$, which are assigned to the $\mathrm{Mo}=\mathrm{O}$ symmetric stretch vibration, $\mathrm{Mo}-\mathrm{O}_{\mathrm{c}}-\mathrm{Mo}$ symmetric bending of edge-shared bridge oxygen and $\mathrm{O}-\mathrm{P}-\mathrm{O}$ vibration, ${ }^{56,57}$ respectively. In addition, the $249 \mathrm{~cm}^{-1}, 164 \mathrm{~cm}^{-1}$ and $107 \mathrm{~cm}^{-1}$ peaks were derived from the $\mathrm{MoO}_{3}$ group of HPMo. ${ }^{58,59}$ The $994 \mathrm{~cm}^{-1}$ and $249 \mathrm{~cm}^{-1}$ peaks of HPMo were also observed in $10 \%, 20 \%$, and $30 \% \mathrm{HPMo} / \mathrm{BN}-\mathrm{IL}$ catalysts, declaring the successful immobilization of HPMo on BN-IL carrier. However, the characteristic peaks of HPMo were not detected on $5 \% \mathrm{HPMo} / \mathrm{BN}-\mathrm{IL}$ catalyst, owing to the lower loadings of HPMo on BN-IL.

\subsection{UV-vis DRS analysis}

Fig. 6 presents the UV-vis DRS spectra of h-BN, $x \%$ HPMo/BN-IL catalysts and HPMo. As seen from Fig. 6a, two obvious signal peaks of h-BN were detected at $209 \mathrm{~nm}$ and $244 \mathrm{~nm}$, and the two peaks also appeared in $5 \%$ and $10 \% \mathrm{HPMo} / \mathrm{BN}-\mathrm{IL}$ catalysts,

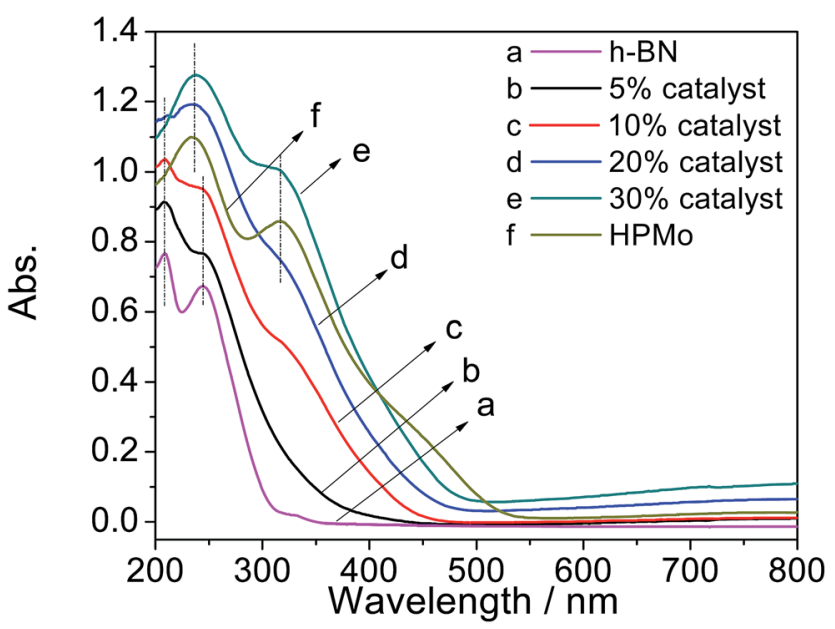

Fig. 6 UV-vis DRS patterns of all catalysts. (a) h-BN; (b) 5\% HPMo/BNIL; (c) 10\% HPMo/BN-IL; (d) 20\% HPMo/BN-IL; (e) 30\% HPMo/BN-IL; (f) HPMo. which suggested that the structure of h-BN was not changed after the introduction of HPMo. For HPMo in Fig. 6f, there were two signal peaks at $233 \mathrm{~nm}$ and $317 \mathrm{~nm}$, attributing to the Keggin structure of heteropoly anion. ${ }^{\mathbf{4 1 , 6 0 , 6 1}}$ Meanwhile, the two signal peaks were also clearly observed in $20 \%$ and $30 \% \mathrm{HPMo} / \mathrm{BN}-\mathrm{IL}$ catalysts, hinting the successful introduction of HPMo into hBN. However, because of the lower loadings of HPMo or the great dispersion of HPMo on BN-IL, the two peaks of HPMo could not be clearly detected in 5\% and 10\% HPMo/BN-IL catalysts. The results are consistent with that in Raman analysis.

\subsection{Nitrogen adsorption/desorption isotherms and pore size distribution curves}

Fig. 7 exhibits the $\mathrm{N}_{2}$ adsorption/desorption isotherms and corresponding pore size distribution curves of h-BN, BN-IL, and $x \%$ HPMo/BN-IL catalysts. As can be seen from Fig. 7a, all samples showed the typical II isotherms with obvious $\mathrm{H}_{3}$ hysteresis loops, and the starting points of hysteresis loops were in the range of $P /$ $P_{0}=0.45-0.55$. The results suggested that the pore structures of h-BN, BN-IL, and $x \%$ HPMo/BN-IL catalysts were all mesoporous structure, which originated from slit-pore of layer material, ${ }^{37}$ and the mesoporous structures were not changed after heterogenization. Compared with h-BN, the adsorption capacities of hysteresis loops in BN-IL and $x \%$ HPMo/BN-IL reduce clearly. The results indicated that the IL and HPMo were successfully immobilized on h-BN, and the introduction of IL and HPMo led to the decrease of the specific surface area for h-BN. The decrease of specific surface areas (SSA) is mainly attributed to following reasons: even though the $\mathrm{h}-\mathrm{BN}$ is an excellent material with a rather high SSA, the grafting of IL may cause the blocking of micropores, which is proved by the pore size distribution curves (Fig. 7b). Additionally, the agglomeration of h-BN is also inevitable because of the existence of long organic ligands on the surface. When HPMo was loaded onto the BN-IL, the SSA further decreased. The decrease of the SSAs mainly origins from the very low SSA of HPMo and the SSA is thusly decreases with the increase of HPMo loading amount.

\subsection{Influence of different catalysts on the removal of 4,6- DMDBT}

Table 1 shows the effects of different catalysts on the removal of 4,6-DMDBT. When only with the HPMo as a catalyst, the
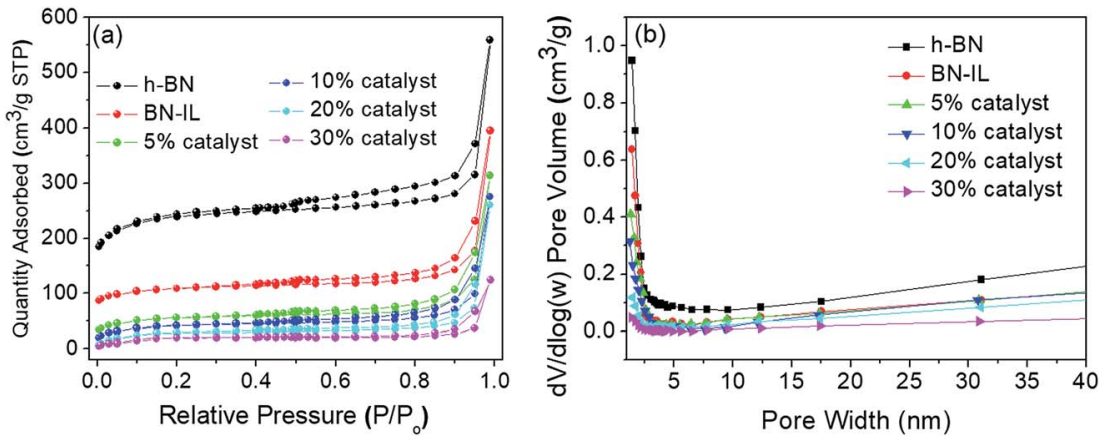

Fig. 7 Nitrogen adsorption-desorption isotherms (a) and pore size distribution curves (b) of h-BN, BN-IL and $x \% \mathrm{HPMo} / \mathrm{BN}-\mathrm{IL}$. 
Table 1 Influence of different catalysts on 4,6-DMDBT

\begin{tabular}{llrl}
\hline & & \multicolumn{2}{c}{ Sulfur removal $(\%)$} \\
\cline { 3 - 4 } Entry & Catalytic systems & Catalyst & Catalyst $+\mathrm{H}_{2} \mathrm{O}_{2}$ \\
\hline $1^{a}$ & HPMo & 8.6 & 14.7 \\
2 & HPMo/BN & 31.5 & 89.1 \\
3 & HPMo/BN-IL & 38.6 & 94.3 \\
4 & HPMo/commercial-BN-IL & 3.1 & 21.4 \\
5 & BN + IL + HPMo & 23.4 & 67.3 \\
6 & h-BN & 32.0 & 32.5
\end{tabular}

${ }^{a}$ This amount is equal with the loadings of HPMo in $20 \%$ HPMo/BN-IL catalyst. Reaction condition: $T=40^{\circ} \mathrm{C} ; n(\mathrm{O}) / n(\mathrm{~S})=4 ; V($ oil $)=5 \mathrm{~mL} ; m$ (catalyst) $=0.05 \mathrm{~g} ; t=100 \mathrm{~min}$.

catalytic activity for 4,6-DMDBT was very low to $8.6 \%$; and even with the $\mathrm{H}_{2} \mathrm{O}_{2}$ as the oxidant, the removal of 4,6-DMDBT was only $14.7 \%$ (entry 1 ). However, when immobilizing HPMo onto h-BN (entry 2), the catalytic activity of HPMo/BN catalyst for 4,6DMDBT reached up to $89.1 \%$, implying the superiority of h-BN as a carrier and the promising activity of HPMo/BN heterogeneous catalyst. Noticeably, with ionic liquid-modified h-BN (BN$\mathrm{IL})$ as a carrier, the catalytic activity of HPMo/BN-IL catalyst (entry 3) improved to 94.3. It is because that the immobilization of IL on h-BN could improve the hydrophobicity of catalyst, leading to a better miscibility among catalyst, oxidant and model oil. As can be noticed in entry 4, when replacing h-BN with commercial-BN, the 4,6-DMDBT removal was only $21.4 \%$. It can be attributed to the great specific surface area and mesoporous structure of h-BN, generating a better dispersion of HPMo on the carrier. Hence, the HPMo/BN-IL is a highefficiency catalyst in ODS field. To verify the importance of high dispersion for HPMo and IL, a simple physical mixing of hBN, IL and HPMo was applied in ODS system (entry 5). The experiment showed that the removal for 4,6-DMDBT was only $67.3 \%$, further illustrating the importance of high dispersion. Obviously, the excellent activity of HPMo/BN-IL for 4,6-DMDBT could be attributed to two aspects: the enhanced hydrophobicity of catalyst induced better miscibility with model oil and the high specific surface area of h-BN. To exclude the possibility of catalytic activity of h-BN, we employed h-BN as a catalyst, the sulfur removal is only $32.50 \%$, close to the adsorptive desulfurization performance of h-BN (entry 6), indicating that the h$\mathrm{BN}$ can only show adsorption performance, which is in favor of the catalytic performance.

\subsection{Effect of loading amounts of HPMo on the removal of 4,6-DMDBT}

The $x \% \mathrm{HPMo} / \mathrm{BN}-\mathrm{IL}$ catalysts were applied into ODS system to investigate the effect of HPMo loading amounts on catalytic activity, and the results are depicted in Fig. 8. As seen from Fig. 8a, when the loading amount of HPMo increased from 5\% to $20 \%$, the removal rates gradually decreased. However, when the HPMo loading further increased to $30 \%$, the catalytic activity descended. To explain the phenomenon, the transmission electron microscope (TEM) image of 30\% HPMo/BN-IL

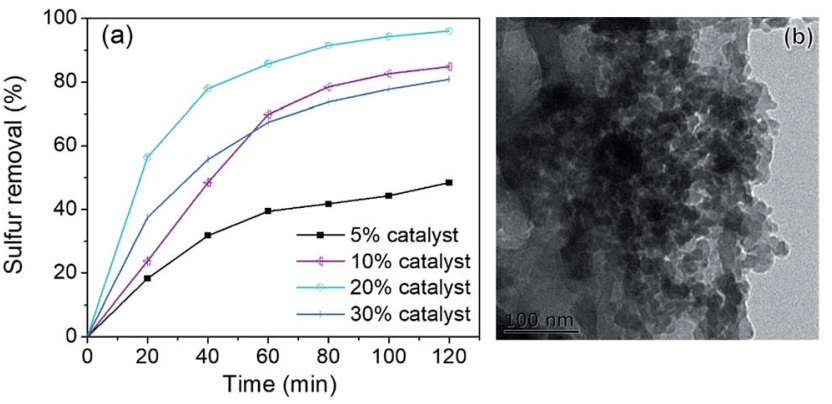

Fig. 8 Effect of different loadings of HPMo on 4,6-DMDBT removal. (a) Sulfur removal of different catalysts; (b) TEM image of $30 \% \mathrm{HPMO} /$ $\mathrm{BN}$-IL. Reaction conditions: $T=40^{\circ} \mathrm{C}, V$ (oil) $=5 \mathrm{~mL}, m$ (catalyst) $=$ $0.05 \mathrm{~g}, n(\mathrm{O}) / n(\mathrm{~S})=4$.

catalysts was recorded in Fig. 8b. In Fig. 8b, an extensive reunion phenomenon of HPMo was observed. It is precisely attributed to that the higher HPMo loading generates aggregation of HPMo on h-BN, which leads to the decrease of specific surface area and the blocking of pore structures, and further hinder the exposure of active sites. The above results are consistent with the results of $\mathrm{N}_{2}$ adsorption/desorption isotherms and pore size distribution curves.

\subsection{Effects of catalyst amounts on the removal of 4,6- DMDBT}

To optimize the catalytic condition, a series of catalytic experiments were performed. Among all the parameters, catalyst amount is one of the most important factors (Fig. 9). As shown in Fig. 9, the removal rates of 4,6-DMDBT increase with the augment of catalyst amounts. When $0.05 \mathrm{~g}$ of catalyst was employed, a $96.07 \%$ of sulfur removal was gained within $120 \mathrm{~min}$. Therefore, $0.05 \mathrm{~g}$ was selected as a more appropriate catalyst amount of HPMo/BN-IL.

\subsection{Effects of temperature and oxidant amounts on oxidation of 4,6-DMDBT}

Effects of reaction temperature and oxidant amounts $(\mathrm{O} / \mathrm{S}$ mole ratio) on oxidizing 4,6-DMDBT were also explored in Fig. 10. As seen from Fig. 10a, the removal rate of 4,6 -DMDBT at $40{ }^{\circ} \mathrm{C}$ is

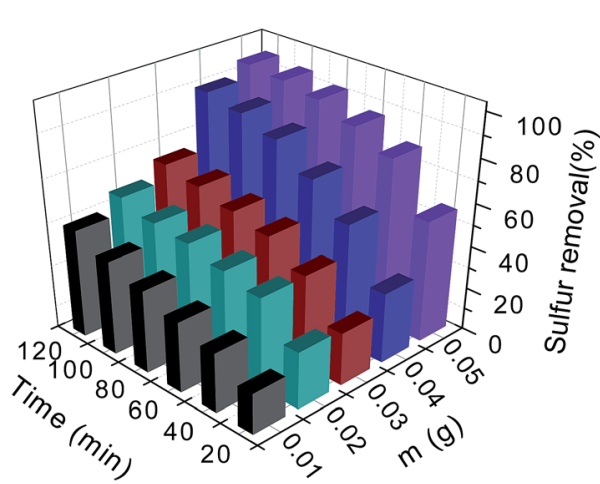

Fig. 9 Effect of different catalyst amounts on oxidation of 4,6DMDBT. Reaction conditions: $T=40^{\circ} \mathrm{C}, V$ (oil) $=5 \mathrm{~mL}, n(\mathrm{O}) / n(\mathrm{~S})=4$. 

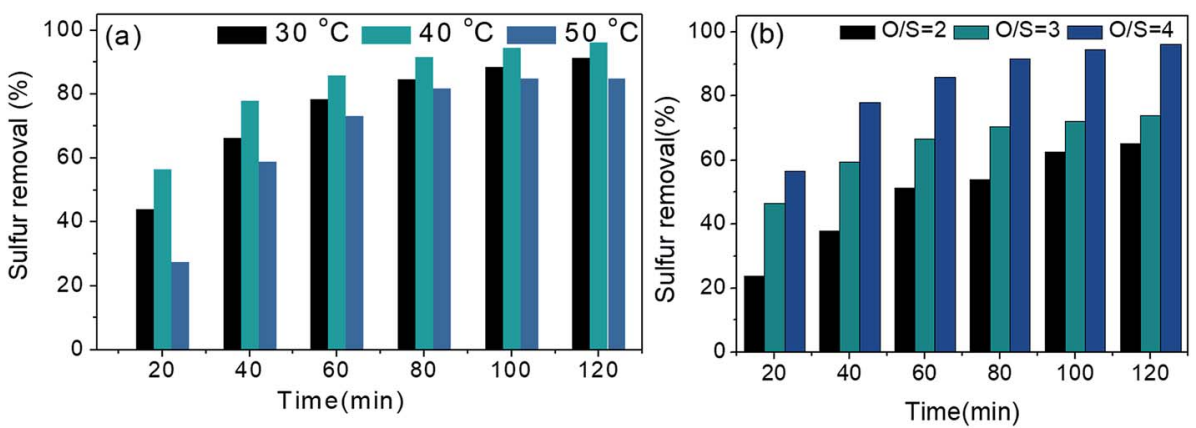

Fig. 10 Effects of (a) reaction temperatures and (b) O/S molar rates on oxidation of 4,6-DMDBT. Reaction conditions: (a) $V$ (oil) $=5 \mathrm{~mL}, \mathrm{~m}$ (20\% catalyst) $=0.05 \mathrm{~g}, n(\mathrm{O}) / n(\mathrm{~S})=4$; (b) $T=40^{\circ} \mathrm{C}, V$ (oil) $=5 \mathrm{~mL}, m(20 \%$ catalyst $)=0.05 \mathrm{~g}$

superior to that at $30{ }^{\circ} \mathrm{C}$. However, when reaction temperature increased to $50{ }^{\circ} \mathrm{C}$, the sulfur removal reduced, owing to that higher temperature can intensify the decomposition of $\mathrm{H}_{2} \mathrm{O}_{2}$ (oxidant), causing the distinct decrease in desulfurization rates. Hence, $40{ }^{\circ} \mathrm{C}$ was selected as the optimum reaction temperature. Fig. $10 \mathrm{~b}$ records the removal effect of $\mathrm{O} / \mathrm{S}$ for oxidation of 4,6-DMDBT. When the O/S mole ratios increased from 2 to 4 , the removal rates of 4,6-DMDBT in $120 \mathrm{~min}$ were $65.09 \%$, $73.8 \%$, and $96.07 \%$, respectively. Therefore, $\mathrm{O} / \mathrm{S}=4$ is considered as the most suitable mole ratio.

\subsection{Effect of different substrates on removal of sulfide}

To explore the catalytic performance of HPMo/BN-IL catalyst for various sulfides in fuel, DBT, 4,6-DMDBT, 3-MBT, and BT were selected as substrates. As presented in Fig. 11, the removals of the four substrates all increased with the augment of reaction time. The removal rates of BT in 120 min was only $27.22 \%$, and it could only up to $31.15 \%$ with another $60 \mathrm{~min}$. However, the catalyst showed remarkable removal effects for 4,6-DMDBT, DBT, and 3MBT. The sulfur removals of 4,6-DMDBT, DBT and 3-MBT can improve to $100 \%, 94.28 \%$ and $84.49 \%$ with 180 min of reaction time, respectively. In oxidative desulfurization process, when considering the effect of substrates on sulfur removal, electron

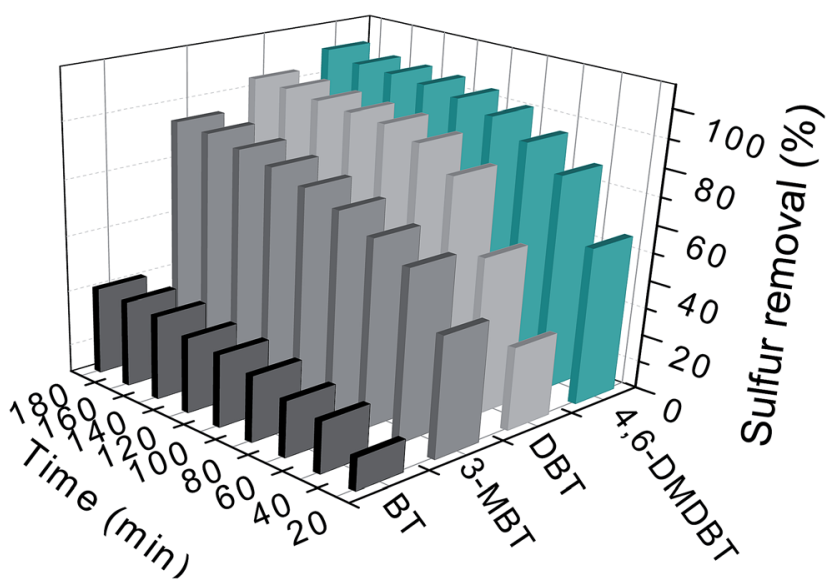

Fig. 11 Oxidizing performance of catalyst on the removal of disparate substrates. Experimental conditions: $T=40^{\circ} \mathrm{C} ; V$ (oil) $=5 \mathrm{~mL} ; m(20 \%$ catalyst $)=0.05 \mathrm{~g} ; n(\mathrm{O}) / n(\mathrm{~S})=4$. structure is a determining factor. When regarding the electron density, it not only just indicates the electron density of sulfur atoms (certainly, it is of great importance), but also means that the aromatic properties of the sulfur compound should be considered. When regarding the electron density of sulfur atoms, the electron densities of $\mathrm{S}$ atoms in 4,6-DMDBT and DBT are respectively 5.623 and $5.576,{ }^{62}$ leading to a higher catalytic activity over 4,6-DMDBT than that over DBT. The electron densities of S atoms in BT and 3-MBT are 5.585 and 5.588, respectively. Thusly, the sulfur removal over BT is lower than that of 3-MBT. However, even though the BT and 3-MBT hold higher S atom electron density, they only have 1 aromatic rings, showing weaker aromatic properties than that of DBT. Both according to our previous reports and considering the catalytic process for heterogeneous catalysis, more aromatic rings of substrates are beneficial to the surface of h-BN based catalysts, which is in favor to the formation of a local high-concentration environment. Thusly, the catalytic activity over DBT is better than those over BT and 3-MBT.

\subsection{Influence of different distractors on oxidation of 4,6- DMDBT}

To explore the desulfurization effects of HPMo/BN-IL catalyst for real oils, different distractors containing model oils were employed and the results are shown in Fig. 12. When adding $10 \mathrm{wt} \%$ of cyclohexene, the sulfur removal showed slightly decrease. Meanwhile, the sulfur removal also decreased with the addition of $10 \mathrm{wt} \%$ of methylbenzene or $10 \mathrm{wt} \%$ of paraxylene. However, with a longer reaction time to $180 \mathrm{~min}$, the sulfur removals in methylbenzene or paraxylene model oil could reach $95.11 \%$ and $93.06 \%$, respectively. The results manifested that the addition of cyclohexene, methylbenzene or paraxylene has little effects on removal rates of 4,6-DMDBT. Afterwards, a mixture of $10 \mathrm{wt} \%$ of cyclohexene, methylbenzene, and paraxylene was added into the 4,6-DMDBT model oil. Fig. 12 illustrates that the sulfur removal of 4,6-DMDBT in a mixed interference oil is significantly lower than that without distractors, but the sulfur removals could still high to $83.05 \%$ with a longer reaction time to $180 \mathrm{~min}$.

\subsection{Recycling performance of HPMo/BN-IL catalyst}

Fig. 13 records the recycling effects of 20\% HPMo/BN-IL catalyst, to detect the stability of catalyst in ODS system. After the reaction, the reaction system stood for a while to separate oil phase and 


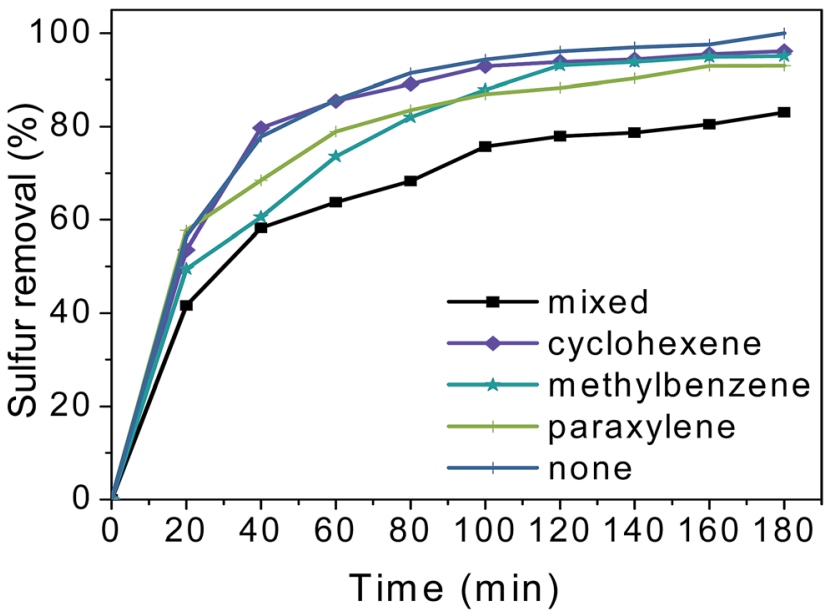

Fig. 12 Influence of different distractors on oxidizing 4,6-DMDBT Reaction conditions: $T=40{ }^{\circ} \mathrm{C} ; V$ (oil) $=5 \mathrm{~mL} ; m$ (20\% catalyst) $=$ $0.05 \mathrm{~g} ; n(\mathrm{O}) / \mathrm{n}(\mathrm{S})=4$.

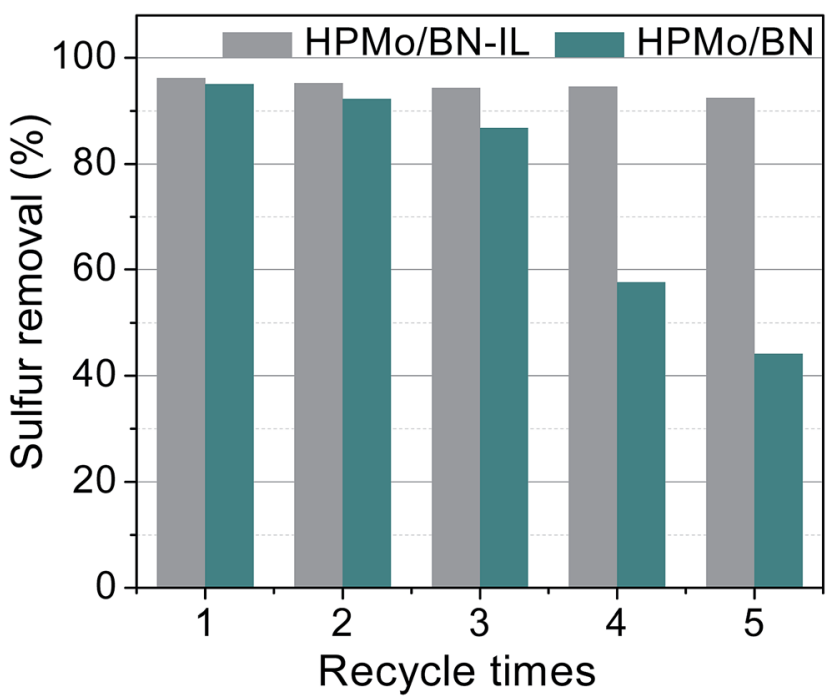

Fig. 13 Recycling performance of HPMo/BN-IL and HPMo/BN catalysts. Reaction conditions: $T=40{ }^{\circ} \mathrm{C} ; \mathrm{V}$ (oil) $=5 \mathrm{~mL} ; m$ (20\% catalyst) $=$ $0.05 \mathrm{~g} ; n(\mathrm{O}) / \mathrm{n}(\mathrm{S})=4$. catalyst phase, then the oil phase was drawn out by a straw. The catalyst phase with a little oil was transferred into an oven for $48 \mathrm{~h}$ under $80{ }^{\circ} \mathrm{C}$ to dry thoroughly the residual oil and $\mathrm{H}_{2} \mathrm{O}_{2}$. Finally, the fresh model oil and $\mathrm{H}_{2} \mathrm{O}_{2}$ were added into the regenerated catalyst for the next run. As shown in Fig. 13, the 20\% HPMo/BNIL catalyst can be recycled for 5 times, and the catalyst activity only decreases $3.7 \%$ after 5 times. The data present the remarkable recyclability and stability of $20 \% \mathrm{HPMo} / \mathrm{BN}-\mathrm{IL}$ catalyst.

In order to further prove the stability of $20 \% \mathrm{HPMo} / \mathrm{BN}-\mathrm{IL}$ catalyst, the recycling activity of $20 \% \mathrm{HPMo} / \mathrm{BN}$ catalyst was also explored by the same methods in Fig. 13. In Fig. 13, the removal rate of 4,6-DMDBT for HPMo/BN-IL catalyst is $92.4 \%$ at 5 times of recycling. However, the sulfur removal of 4,6-DMDBT for $\mathrm{HPMo} / \mathrm{BN}$ drastically decreased to $\sim 42 \%$ of sulfur removal after 5 times of recycling. Therefore, the introduction of IL can improve the stability of HPMo/BN-IL catalyst, making active centers more stable.

\subsection{Speculation of catalytic oxidative mechanism}

The residual sulfides and reaction products of HPMo/BN-IL system were detected by gas chromatography-mass spectrometry (GC-MS). The detection steps are as follows: after ODS reactions, the reaction liquid was centrifuged to separate upper oil phase and catalyst phase. The upper oil phase was directly detected by GC-MS to analyze residual sulfides and reaction products. While the catalyst phase was re-extracted by $\mathrm{CCl} 4$ for further analysis. The GC-MS analysis is shown in Fig. 14. Fig. 14A is the GC-MS spectrogram of the oil phase, the 4,6-DMDBT was detected in oil phase both at $60 \mathrm{~min}$ (Fig. 14a-A) and after reaction (Fig. 14a-B), but the peak of 4,6-DMDBT after the reaction is weaker than that at $60 \mathrm{~min}$. Fig. 14B is the GC-MS spectrogram of catalyst phase, as depicted in Fig. 14B, the 4,6-DMDBT and its product of 4,6-DMDBTO ${ }_{2}$ were observed in catalyst phase at $60 \mathrm{~min}$ (Fig. 14b-A) and after reaction (Fig. 14b-B). The result showed that 4,6-DMDBT can be readily adsorbed onto catalysts phase and further oxidized to sulfone for separation.

Fig. 15 describes the proposed ODS process of HPMo/BN-IL system, and the specific desulfurization process is as follows: first, 4,6-DMDBT and $\mathrm{H}_{2} \mathrm{O}_{2}$ are adsorbed on the surface of catalyst, then $\mathrm{H}_{2} \mathrm{O}_{2}$ provides reactive oxygen to $\mathrm{Mo}=\mathrm{O}$ groups to
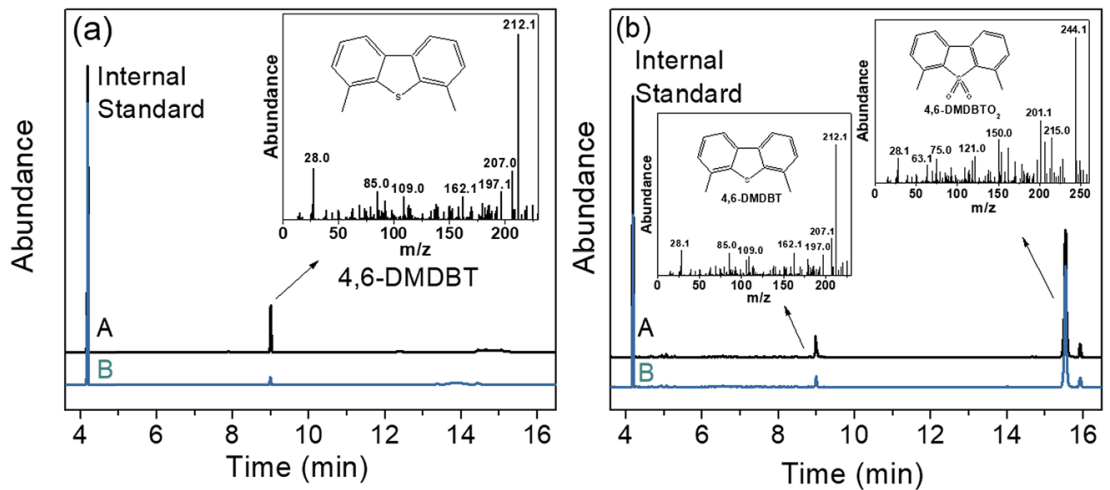

Fig. 14 GC-MS spectrograms of ODS process (a) oil phase (A) in 60 min and (B) after reaction; (b) catalyst phase (A) in 60 min and (B) after the reaction 


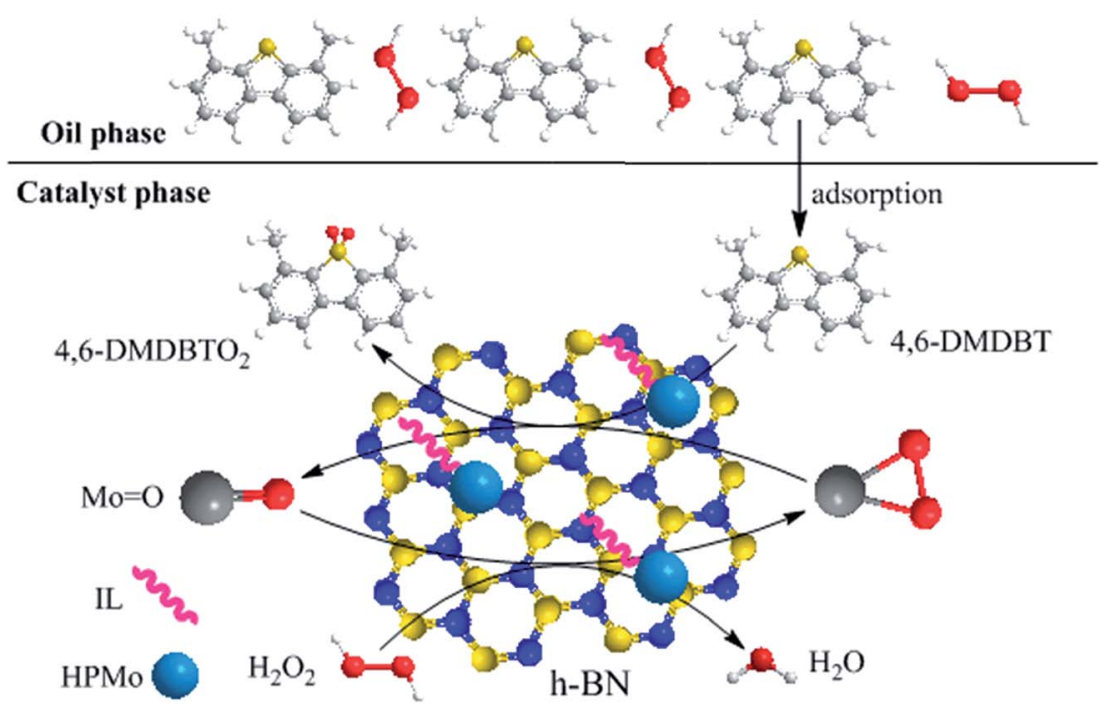

Fig. 15 The proposed mechanism of HPMo/BN-IL system to removal 4,6-DMDBT.

form oxygen species $\mathrm{Mo}(\mathrm{O})_{2},{ }^{63,64}$ and sulfide is oxidized into 4,6$\mathrm{DMDBTO}_{2}$ by $\mathrm{Mo}(\mathrm{O})_{2}$ to realize desulfurization. Once the 4,6DMDBT is oxidized into 4,6-DMDBTO ${ }_{2}$, the catalyst will adsorb sulfides in model oil to keep local high concentration.

\section{Conclusions}

In summary, the HPMo is immobilized on the ionic liquidmodified h-BN (BN-IL) to prepare HPMo/BN-IL heterogeneous catalysts. The immobilization process can not only generate a great dispersion of HPMo but also make the active sites more stable. HPMo/BN-IL system showed excellent catalytic activity for DBT, 4,6-DMDBT, and 3-MBT, which can be attributed to the specific surface areas of h-BN, great dispersion of HPMo and unique porous structure. And the introduction of IL can improve the stability of catalyst, which makes HPMo/BN-IL catalyst show better recyclability than HPMo/BN. Reaction conditions, influences of interferents, reaction products, and proposed reaction process are researched in detail.

\section{Conflicts of interest}

There are no conflicts to declare.

\section{Acknowledgements}

This current work is financially supported by the National Natural Science Foundation of China (No. 21576122, 21507046, 21506083), Six Big Talent Peak in Jiangsu province (JNHB-004), Jiangsu Key Lab of Material Tribology Foundation (No. Kjsmcx201303), University Graduate Student Research and Creative Project of Jiangsu (No. SJLX15_0488).

\section{References}

1 H. Zhao and G. A. Baker, Front. Chem. Sci. Eng., 2015, 9, 262279.
2 W. S. Zhu, C. Wang, H. P. Li, P. W. Wu, S. H. Xun, W. Jiang, Z. G. Chen, Z. Zhao and H. M. Li, Green Chem., 2015, 17, 2464-2472.

3 R. Menzel, D. Iruretagoyena, Y. Wang, S. M. Bawaked, M. Mokhtar, S. A. Al-Thabaiti, S. N. Basahel and M. S. P. Shaffer, Fuel, 2016, 181, 531-536.

4 X. Y. Zeng, X. Y. Xiao, Y. Li, J. Y. Chen and H. L. Wang, Appl. Catal., B, 2017, 209, 98-109.

5 X. X. Zhang, W. Zhang, D. Tian, Z. H. Zhou and C. H. Lu, RSC Adv., 2013, 3, 7722.

6 D. H. Wang, N. Liu, J. Y. Zhang, X. Zhao, W. H. Zhang and M. H. Zhang, J. Mol. Catal. A: Chem., 2014, 393, 47-55.

7 J. L. García-Gutiérrez, G. C. Laredo, P. García-Gutiérrez and F. Jiménez-Cruz, Fuel, 2014, 138, 118-125.

8 J. J. Yuan, J. Xiong, J. H. Wang, W. J. Ding, L. Yang, M. Zhang, W. S. Zhu and H. M. Li, J. Porous Mater., 2016, 23, 823-831.

9 H. Xu, Z. Han, D. J. Zhang and C. B. Liu, J. Mol. Catal. A: Chem., 2015, 398, 297-303.

10 M. Li, M. Zhang, A. M. Wei, W. S. Zhu, S. H. Xun, Y. N. Li, H. P. Li and H. M. Li, J. Mol. Catal. A: Chem., 2015, 406, 23-30.

11 P. W. Wu, S. Z. Yang, W. S. Zhu, H. P. Li, Y. H. Chao, H. Y. Zhu, H. M. Li and S. Dai, Small, 2017, 1701857.

12 C. M. Granadeiro, S. O. Ribeiro, M. Karmaoui, R. Valenca, J. C. Ribeiro, B. de Castro, L. Cunha-Silva and S. S. Balula, Chem. Commun., 2015, 51, 13818-13821.

13 H. Y. Lü, P. C. Li, C. L. Deng, W. Z. Ren, S. N. Wang, P. Liu and H. Zhang, Chem. Commun., 2015, 51, 10703-10706.

14 P. W. Wu, W. S. Zhu, A. M. Wei, B. L. Dai, Y. H. Chao, C. F. Li, H. M. Li and S. Dai, Chem.-Eur. J., 2015, 21, 15421-15427.

15 W. J. Ding, W. S. Zhu, J. Xiong, L. Yang, A. M. Wei, M. Zhang and H. M. Li, Chem. Eng. J., 2015, 266, 213-221.

16 C. X. Shi, W. X. Wang, N. Liu, X. Y. Xu, D. H. Wang, M. H. Zhang, P. C. Sun and T. H. Chen, Chem. Commun., 2015, 51, 11500-11503. 
17 M. Chamack, A. R. Mahjoub and H. Aghayan, Chem. Eng. J., 2014, 255, 686-694.

18 F. L. Yu, C. Y. Liu, B. Yuan, C. X. Xie and S. T. Yu, Catal. Commun., 2015, 68, 49-52.

19 F. L. Yu, Q. Y. Wang, B. Yuan, C. X. Xie and S. T. Yu, Chem. Eng. J., 2017, 309, 298-304.

20 M. Kooti and E. Nasiri, J. Mol. Catal. A: Chem., 2015, 406, 168-177.

21 Y. Chen, Y. Cao, Y. Suo, G. P. Zheng, X. X. Guan and X. C. Zheng, J. Taiwan Inst. Chem. Eng., 2015, 51, 186-192.

22 K. V. Avramidou, F. Zaccheria, S. A. Karakoulia, K. S. Triantafyllidis and N. Ravasio, Mol. Catal., 2017, 439, 60-71.

23 D. P. Sawant, J. Justus, V. V. Balasubramanian, K. Ariga, P. Srinivasu, S. Velmathi, S. B. Halligudi and A. Vinu, Chem.-Eur. J., 2008, 14, 3200-3212.

24 N. Songsiri, G. L. Rempel and P. Prasassarakich, Mol. Catal., 2017, 439, 41-49.

25 Y. Cao, M. R. Lu, J. H. Fang, L. Y. Shi and D. S. Zhang, Chem. Commun., 2017, 53, 7549-7552.

26 M. T. Li, W. S. Zhu, P. F. Zhang, Y. H. Chao, Q. He, B. L. Yang, H. M. Li, A. Borisevich and S. Dai, Small, 2016, 12, 3535-3542.

27 T. T. Li, L. J. Wang, K. Zhang, Y. C. Xu, X. Y. Long, S. J. Gao, R. Li and Y. G. Yao, Small, 2016, 12, 4960-4965.

28 J. J. Liu, R. G. Kutty, Q. S. Zheng, V. Eswariah, S. Sreejith and Z. Liu, Small, 2017, 13, 1602456.

29 W. S. Zhu, Z. L. Wu, G. S. Foo, X. Gao, M. X. Zhou, B. Liu, G. M. Veith, P. W. Wu, K. L. Browning, H. N. Lee, H. M. Li, S. Dai and H. Y. Zhu, Nat. Commun., 2017, 8, 15291.

30 P. W. Wu, W. S. Zhu, Y. H. Chao, J. S. Zhang, P. F. Zhang, H. Y. Zhu, C. F. Li, Z. G. Chen, H. M. Li and S. Dai, Chem. Commun., 2016, 52, 144-147.

31 J. Yin, J. Yu, X. M. Li, J. D. Li, J. X. Zhou, Z. H. Zhang and W. L. Guo, Small, 2015, 11, 4497-4502.

32 C. J. Huang, C. Chen, X. X. Ye, W. Q. Ye, J. L. Hu, C. Xu and X. Q. Qiu, J. Mater. Chem. A, 2013, 1, 12192-12197.

33 C. J. Huang, W. Q. Ye, Q. W. Liu and X. Q. Qiu, ACS Appl. Mater. Interfaces, 2014, 6, 14469-14476.

34 H. Y. Zhu, Z. L. Wu, D. Su, G. M. Veith, H. F. Lu, P. F. Zhang, S. H. Chai and S. Dai, J. Am. Chem. Soc., 2015, 137, 1015610159.

35 H. Y. Zhu, S. Zhang, Y. X. Huang, L. H. Wu and S. H. Sun, Nano Lett., 2013, 13, 2947-2951.

36 W. S. Zhu, B. L. Dai, P. W. Wu, Y. H. Chao, J. Xiong, S. H. Xun, H. P. Li and H. M. Li, ACS Sustainable Chem. Eng., 2015, 3, 186-194.

37 H. Y. Ji, J. Sun, P. W. Wu, B. L. Dai, Y. H. Chao, M. Zhang, W. Jiang, W. S. Zhu and H. M. Li, J. Mol. Catal. A: Chem., 2016, 423, 207-215.

38 B. L. Dai, P. W. Wu, W. S. Zhu, Y. H. Chao, J. Sun, J. Xiong, W. Jiang and H. M. Li, RSC Adv., 2016, 6, 140-147.

39 C. C. Tang, Y. Bando, T. Sato and K. Kurashima, Adv. Mater., 2002, 14, 1046-1049.

40 J. Xiong, W. S. Zhu, W. J. Ding, L. Yang, Y. H. Chao, H. P. Li, F. X. Zhu and H. M. Li, Ind. Eng. Chem. Res., 2014, 53, 1989519904.
41 W. S. Zhu, W. L. Huang, H. M. Li, M. Zhang, W. Jiang, G. Y. Chen and C. R. Han, Fuel Process. Technol., 2011, 92, 1842-1848.

42 F. J. Méndez, A. Llanos, M. Echeverría, R. Jáuregui, Y. Villasana, Y. Díaz, G. Liendo-Polanco, M. A. RamosGarcía, T. Zoltan and J. L. Brito, Fuel, 2013, 110, 249-258.

43 J. H. Qiu, G. H. Wang, D. L. Zeng, Y. Tang, M. Wang and Y. J. Li, Fuel Process. Technol., 2009, 90, 1538-1542.

44 W. W. Lei, D. Portehault, R. Dimova and M. Antoniettit, J. Am. Chem. Soc., 2011, 133, 7121-7127.

45 F. J. Liu, L. Wang, Q. Sun, L. F. Zhu, X. J. Meng and F. S. Xiao, J. Am. Chem. Soc., 2012, 134, 16948-16950.

46 X. J. Wang, W. Y. Yang, F. T. Li, J. Zhao, R. H. Liu, S. J. Liu and B. Li, J. Hazard. Mater., 2015, 292, 126-136.

47 Z. Ai, W. Ho, S. Lee and L. Zhang, Environ. Sci. Technol., 2009, 43, 4143-4150.

48 W. Jiang, D. Zheng, S. H. Xun, Y. J. Qin, Q. Q. Lu, W. S. Zhu and H. M. Li, Fuel, 2017, 190, 1-9.

49 T. Sainsbury, A. Satti, P. May, Z. M. Wang, I. McGovern, Y. K. Gun'ko and J. Coleman, J. Am. Chem. Soc., 2012, 134, 18758-18771.

50 W. W. Lei, D. Portehault, D. Liu, S. Qin and Y. Chen, Nat. Commun., 2013, 4, 1777.

51 M. Nakayama, T. Ii and K. Ogura, J. Mater. Res., 2003, 18, 2509-2514.

52 X. J. Song, W. C. Zhu, K. G. Li, J. Wang, H. L. Niu, H. C. Gao, W. Gao, W. X. Zhang, J. H. Yu and M. J. Jia, Catal. Today, 2016, 259, 59-65.

53 I. Tamiolakis, I. N. Lykakis, A. P. Katsoulidis, M. Stratakis and G. S. Armatas, Chem. Mater., 2011, 23, 4204-4211.

54 W. Jiang, D. Zheng, S. Xun, Y. Qin, Q. Lu, W. Zhu and H. Li, Fuel, 2017, 190, 1-7.

55 Y. Kubota, K. Watanabe, O. Tsuda and T. Taniguchi, Science, 2007, 317, 932-934.

56 V. Balaga, J. Pedada, H. B. Friedrich and S. Singh, J. Mol. Catal. A: Chem., 2016, 425, 116-123.

57 B. Viswanadham, J. Pedada, H. B. Friedrich and S. Singh, Catal. Lett., 2016, 146, 1470-1477.

58 B. Viswanadham, A. Srikanth and K. V. R. Chary, J. Chem. Sci., 2014, 126, 445-454.

59 B. Viswanadham, P. Jhansi, K. V. R. Chary, H. B. Friedrich and S. Singh, Catal. Lett., 2015, 146, 364-372.

60 H. Aliyan, R. Fazaeli and N. Habibollahi, J. Korean Chem. Soc., 2012, 56, 591-596.

61 Y. Ding, B. C. Ma, Q. Gao, G. X. Li, L. Yan and J. S. Suo, J. Mol. Catal. A: Chem., 2005, 230, 121-128.

62 H. P. Li, W. S. Zhu, S. W. Zhu, J. X. Xia, Y. H. Chang, W. Jiang, M. Zhang, Y. W. Zhou and H. M. Li, AIChE J., 2016, 62, 20872100.

63 J. Zhang, A. Wang, Y. J. Wang, H. Y. Wang and J. Z. Gui, Chem. Eng. J., 2014, 245, 65-70.

64 J. L. García-Gutiérrez, G. A. Fuentes, M. E. Hernández-Terán, P. García, F. Murrieta-Guevara and F. Jiménez-Cruz, Appl. Catal., A, 2008, 334, 366-373. 